\title{
Invariances in Physics and Group Theory
}

\author{
Jean-Bernard Zuber \\ LPTHE, CNRS-UMR 7589 and Université Pierre et Marie Curie, \\ 4 place Jussieu, 75252 Paris Cedex 5, France \\ email:zuber@lpthe.jussieu.fr
}

\begin{abstract}
This is a short review of the heritage of Klein's Erlangen program in modern physics 1

2000 Mathematics Subject Classification: 20G45; 20H15; 22E70; 70S10; 70S15; 8103;

81Rxx; 81Txx; 8303;

Keywords: Lie groups; representation theory; invariances; quantum field theories; quantum symmetries
\end{abstract}

\section{Contents}

1 Introduction ............................ 1

2 Early group theory in 19th century physics: crystallography . . . . 2

3 Special Relativity and Lorentz group: Lorentz, Poincaré, Einstein ... 4

4 General Relativity... and gauge theories . . . . . . . . . . . 5

5 Emmy Noether: invariances and conservation laws . . . . . . . . 6

6 Invariances in Quantum Mechanics . . . . . . . . . . . . . . . 9

7 Invariances in particle physics . . . . . . . . . . . . . . . . 10

8 The many implementations of symmetries in the quantum world . . 12

9 Conclusions . . . . . . . . . . . . . . . . . 17

\section{Introduction}

Let us be honest: most physicists of our time, even theorists, do not have a very clear notion of what Klein's Erlanger Programm is about, and this is an

\footnotetext{
${ }^{1}$ Talk given at the Conference Lie and Klein; the Erlangen program and its impact on mathematics and physics, Strasbourg, Sept. 2012
} 
understatement... If we read Weyl 31], however, “According to Klein's Erlanger Program any geometry of a point-field is based on a particular transformation group $\mathfrak{G}$ of the field; figures which are equivalent with respect to $\mathfrak{G}$, and which can therefore be carried into one another by a transformation of $\mathfrak{G}$, are to be considered as the same..."

and substitute "physical systems" for "figures", we see that modern physicists, like Molière's character Monsieur Jourdain, who was delighted to learn that he had been speaking prose all his life without knowing, would love to hear that they keep following Klein's program...

The aim of this lecture is indeed to illustrate how group theory associated with invariances of the geometry or the dynamics of a physical system has pervaded all modern physics and has become of everyday use in the physicist's toolbox. A word of caution, though. As the author of these lines is not a professional historian of science, this lecture will undoubtedly present only a biased and incomplete view of this vast subject.

\section{Early group theory in 19th century physics: crystallography}

Before the birth of Lie group theory and Klein's Erlangen program, physicists had realized the role of symmetry in Nature and foreseen the importance of group theory in physical sciences. It had been known for long by artists that 2-dimensional periodic patterns - tilings or wall paper motives, i.e. twodimensional "crystals" - were coming in finite types. There are 17 types of symmetry - 17 space groups in modern terminology - in two dimensions. For a beautiful illustration, see the web sitehttp://en.wikipedia.org/wiki/Wallpaper_group. Crystallographers then set out to tabulate the corresponding structures in 3 dimensions, classifying in turn the point groups, i.e. groups that fix a point of a lattice, the classes of lattices, and the space groups, taking translations into account. This long endeavour kept them busy for the major part of the nineteenth century, with important steps achieved by Frankenheim and by Hessel (32 point groups in 3 dimensions, in the 1830's), by Bravais (14 classes of lattices, circa 1850), C. Jordan (who emphasized the role of groups), and many others. The program was completed in the early nineties of that century, by Schönflies2, Fedorov and Barlow (1891-94), with the classification of the 230 space groups in 3 dimensions, see [2, 21, 12, 16]. The situation is summarized in the following table

\footnotetext{
${ }^{2}$ It may be relevant to observe that Schönflies was directed to this problem by Klein, who saw it as a nice illustration of his program...
} 


\begin{tabular}{|c|c|c|c|}
\hline Dimension $d$ & Point Groups & Lattices & Space groups \\
\hline$d=1$ & 2 & 1 & 7 \\
$d=2$ & 10 & 5 & 17 \\
$d=3$ & 32 & 14 & 230 \\
\hline
\end{tabular}

According to H. Weyl 31] "The most important application of group theory to natural science heretofore has been in this field". It is interesting to notice that Weyl wrote this comment in 1928, many years after the birth of Relativity - both Special and General -, and as he was himself working on the applications of group theory to quantum physics...

Breaking of symmetry. If it is important in physical sciences to know the possible types of symmetry, it is maybe even more interesting to understand the way these symmetries may be broken 3 . This was emphasized in a particularly clear way by Pierre Curie, as stated in his principle (1894) [3]: "Elements of symmetry of causes must be found in effects; when some effects reveal some asymmetry, that asymmetry must be found in causes." Or in a more cursive way: "C'est la dissymétrie qui crée le phénomène".

An example is provided by the phenomenon of piezoelectricity, i.e. the creation of an electric (vector) field $E$ in a crystalline material subject to a mechanical stress. The latter is described by a rank-two tensor $u$, in a linear approximation the electric field is proportional to $u, E_{i}=\sum_{j k} \gamma_{i, j k} u_{j k}$, and hence the phenomenon depends on the existence of a non vanishing rank 3 tensor $\gamma_{i, j k} \neq 0$; if the crystal admits a symmetry by "inversion", (i.e. reflection with respect to a point), $\gamma_{i, j k}$ is changed into $-\gamma_{i, j k}$ under inversion and must vanish, and this rules out piezoelectricity in many crystal classes. Only non symmetric crystalline classes may give rise to piezoelectricity.

Curie also understood that breaking of a symmetry under a group $G$ may leave invariance under a subgroup (an "intergroupe" in his terms) $H$ of $G$, an idea still quite topical. For instance he classified the possible breakings and subgroups of a system invariant under rotations around an axis, i.e. under the group $D_{\infty}$ in modern terminology.

Limits of group theory. As noticed by M. Senechal 23, "group theory cannot answer a question that seems fundamental today: which shapes tile space and in what way?". That question has of course become highly relevant since the discovery some 30 years ago of quasicrystals. In this new class of materials, rotational order does not extend to large distances and translation invariance is lost. Still diffraction of X-rays leads to patterns of bright spots exhibiting some symmetry. This has led the International Union of Crystallography to redefine the term "crystal" so as to include both ordinary periodic crystals and quasicrystals. According to this new definition, a crystal is "any solid having an essentially discrete diffraction diagram".

\footnotetext{
${ }^{3}$ This is also an interesting issue in art, see 33 .
} 


\section{Special Relativity and Lorentz group: Lorentz, Poincaré, Einstein ...}

Special Relativity is often regarded as the first appearance of Lie group theory in modern physics. Let us recall some of the crucial steps, referring the reader to more scholarly sources [18, 4, for further details.

- Lorentz (1892-1904) (after Voigt and FitzGerald, and in parallel to Larmor) discovers what are now called the Lorentz transformations and the resulting contraction of lengths. His purpose is to make the MichelsonMorley experiment consistent with the existence of aether.

- Poincaré (1905) establishes the covariance of Maxwell equations under Lorentz transformations; he also sees that Lorentz transformations together with space rotations leave the form $x \cdot x:=x^{2}+y^{2}+z^{2}-c^{2} t^{2}$ invariant and form a group, thus giving them their proper geometric meaning, much in the spirit of Klein. In his approach, however, the Lorentz group is not derived from first principles.

- Einstein (1905) starting from two principles - (i) the principle of relativity: physical laws do not depend on the inertial frame of the observer; and (ii) in an inertial frame the speed of light $c$ is an absolute constant of Physics, independent of the uniform motion of the source - constructs the Lorentz transformations; he notices as a side remark that Lorentz special transformations (or "boosts" as we call them now) of colinear velocities form a group, "wie die sein muss" (as they should) 4 ; he proves that they leave Maxwell's equations invariant, but does not seem to notice or at least does not comment on the fact that they also preserve the form $x \cdot x$.

- Minkowski (1908) introduces "space-time", identifies the Lorentz group as the invariance group of the metric $x_{1}^{2}+x_{2}^{2}+x_{3}^{2}+x_{4}^{2}$ with $x_{4}=i c t$, makes use of the notion of 4 -vectors and tensors, and shows the covariant way of writing Maxwell equations. At first Einstein is not impressed by this piece of work, qualifying it as "überflüssige Gelehrsamkeit" (superfluous erudition)! 6]. After starting to work on gravitation, however, Einstein soon realizes the power of tensor methods...

Thus, although Einstein made a real breakthrough in physics and utterly changed our view of space and time by "propounding a new chronogeometry" [4, it seems fair to say that group theory played a very minor role in his work and his lines of thought.

More mathematically inclined people thought otherwise. We have already mentioned Poincaré's and Minkowski's works. Klein (1910) [14] observes: "One could replace 'theory of invariants relative to a group of transformations'

\footnotetext{
${ }^{4}$ the only occurrence of the word "Gruppe" in his paper...
} 
by the words 'relativity theory with respect to a group'." For him, Galilean relativity or Special Relativity were clearly in the straight line of his Program.

\section{General Relativity... and gauge theories}

General Relativity is an emblematic case illustrating Klein's program in a differential geometric context. There, following Einstein's vision, one postulates the invariance of the equations of the gravitational field under general coordinate transformations. And by a sort of reverse engineering, one looks for equations knowing the invariance group. This is what was achieved by Einstein and by Hilbert (1915), with the celebrated equation

$$
R_{\mu \nu}-\frac{1}{2} R g_{\mu \nu}=\kappa T_{\mu \nu}
$$

with $R_{\mu \nu}$ the Ricci tensor, $R=R_{\mu}^{\mu}$ its curvature, $T_{\mu \nu}$ the energy-momentum tensor, and $\kappa=\frac{8 \pi G}{c^{4}}$ where $G$ is Newton's gravitational constant. Recall that Hilbert derived this equation from the invariant action

$$
S=\int\left[\frac{1}{2 \kappa} R+\mathcal{L}_{\mathrm{M}}\right] \sqrt{-g} d^{4} x,
$$

with $\mathcal{L}_{\mathrm{M}}$ describing the invariant coupling of gravity to matter, and $T_{\mu \nu}=$ $\partial\left(\mathcal{L}_{\mathrm{M}} \sqrt{-g}\right) / \partial g^{\mu \nu}$. I shall not dwell more on that subject, as it was treated by other contributors to the meeting.

Let me rather make a big leap forward in time, and observe that a similar approach was taken in the construction of non-abelian gauge theories. The gauge invariance of electrodynamics had been observed by Weyl (1918) and reformulated later by him into what we now call U(1) gauge invariance 30, 32 . Looking for a generalization to non abelian groups $G$, i.e. postulating invariance under a certain infinite dimensional group of local, space-time dependent transformations, Yang and Mills (1954) 39] were led to an (essentially) unique solution, with Lagrangian density

$$
\mathcal{L}=\frac{1}{2 g^{2}} \operatorname{tr} F_{\mu \nu} F^{\mu \nu}, \quad F_{\mu \nu}=\partial_{\mu} A_{\nu}-\partial_{\nu} A_{\mu}-\left[A_{\mu}, A_{\nu}\right],
$$

with the gauge field $A_{\mu}$ and its field strength tensor $F_{\mu \nu}$ (a connection and its curvature on a fiber bundle) taking values in the Lie algebra of $G$ or one of its representations. Here and below, $\partial_{\mu}$ stands for $\frac{\partial}{\partial x^{\mu}}$. $\mathcal{L}$ is invariant under local infinitesimal changes $\delta A_{\mu}(x)=D_{\mu} \delta \alpha(x)$, with $D_{\mu}=\partial_{\mu}-\left[A_{\mu}, \cdot\right]$ the covariant derivative, and $\delta \alpha \in \operatorname{Lie} G$. A term $\mathcal{L}_{\mathrm{m}}$ may then be added to $\mathcal{L}$ to describe the gauge invariant coupling to matter. This now famous and ubiquitous YangMills theory is the cornerstone of the Standard Model of particle physics, see below. 
To summarize, here are two cases (Einstein-Hilbert, Yang-Mills) in which invariances and geometry of space (either real space-time or "internal" space) prescribe the dynamics. According to Yang's motto 38, "symmetry dictates interaction".

\section{Emmy Noether: invariances and conservation laws}

Noether's celebrated paper (1918) 17, presented on the occasion of Klein's academic Jubilee, contains two theorems on group invariance in variational problems. I give a sketch of her results, using modern terminology and notations, and I refer to [15] for a translation of her original article and a detailed and critical reading, see also 13 .

Consider a field theory described by an action principle in a, say, 4-dimensional space-time with coordinates $x=\left(\vec{x}, t \equiv x^{0}\right)$

$$
S=\int \mathcal{L}\left(x ; \phi^{i}(x), \partial \phi^{i}(x), \cdots\right) d^{4} x
$$

with $S$ the action and $\mathcal{L}$ the Lagrangian density, a local function of a collection of fields $\left\{\phi^{i}\right\}$ and of finitely many of their derivatives. Assume the invariance of $\mathcal{L} d^{4} x$ (and hence of $S$ ) under a Lie group of coordinate and field variations $x \mapsto x^{\prime}, \phi \mapsto \phi^{\prime}$. Then Noether's first theorem asserts:

Theorem 5.1 (Noether). An $n$ dimensional Lie group of invariance of $\mathcal{L} d^{4} x$ implies the existence of $n$ independent divergenceless currents

$j_{s}^{\mu}=\left(j_{s}^{0}(\vec{x}, t), \vec{j}_{s}(\vec{x}, t)\right), \quad$ i.e. $\quad \partial_{\mu} j_{s}^{\mu} \equiv \frac{\partial}{\partial t} j_{s}^{0}-\operatorname{div} \vec{j}_{s}=0, \quad s=1, \cdots, n$,

from which, by Stokes theorem, $n$ independent conservation laws follow

$$
\frac{d}{d t} Q_{s}:=\frac{d}{d t} \int d^{3} x j_{s}^{0}(\vec{x}, t)=\int d^{3} x \operatorname{div} \vec{j}_{s}(\vec{x}, t)=0 .
$$

(The currents are assumed to vanish fast enough at spatial infinity to justify the last step.) Suppose that $\mathcal{L}$ depends only on $\phi$ and its first derivatives $\partial \phi$. Write coordinate and field infinitesimal variations as $\delta x^{\mu}=X_{s}^{\mu}(x, \phi) \delta a^{s}$ and $\delta \phi^{i}=Z_{s}^{i}(x, \phi) \delta a^{s}$, where $a^{s}, s=1, \cdots n$, are parameters in the Lie algebra, and Einstein's convention of summation over repeated indices is used. Then 
one finds

$$
\begin{aligned}
j_{s}^{\mu} & =-\frac{\partial \mathcal{L}}{\partial \partial_{\mu} \phi^{i}}\left(Z_{s}^{i}-\partial_{\rho} \phi^{i} X_{s}^{\rho}\right)-X_{s}^{\mu} \mathcal{L} \\
\partial_{\mu} j_{s}^{\mu} \delta a^{s} & =\sum_{i} \Psi_{i} \delta \phi^{i} \quad \text { where } \Psi_{i}:=\frac{\delta \mathcal{L}}{\delta \phi^{i}}:=\frac{\partial \mathcal{L}}{\partial \phi^{i}}-\partial_{\mu} \frac{\partial \mathcal{L}}{\partial \partial_{\mu} \phi^{i}} \\
& =0 \text { by Euler - Lagrange equations } .
\end{aligned}
$$

In Noether's paper, the converse property, namely that conservation laws imply invariance, is also derived. This first theorem was subsequently generalized by Bessel-Hagen (1921) to the case where $\mathcal{L} d^{4} x$ is invariant up to a total divergence $\delta a^{s} \partial_{\mu} k_{s}^{\mu} d^{4} x$, in which case $j_{s}^{\mu}$ is just modified by the additional term $k_{s}^{\mu}$.

As an example, consider a theory involving a complex scalar field $\phi$ with Lagrangian $\mathcal{L}=\partial_{\mu} \phi^{*} \partial^{\mu} \phi-V\left(\phi^{*} \phi\right), V$ some arbitrary polynomial potential. The Lagrangian is invariant under the U(1) group of transformations $\phi(x) \rightarrow$ $e^{i \alpha} \phi(x)$, leading to a conserved Noether current $j_{\mu}(x)=i\left(\phi^{*}(x) \partial_{\mu} \phi(x)-\right.$ $\left.\left(\partial_{\mu} \phi(x)\right)^{*} \phi(x)\right)$. The associated conserved $\mathrm{U}(1)$ charge may be thought of as an electric (or baryonic or leptonic...) charge.

Thus Noether's first theorem establishes a link between invariances under continuous transformations and conservation laws. This was not a new result in physics. There had been early precursors: Lagrange (1811), Hamilton (1834), Jacobi (1837) had uncovered the fundamental conservation laws of energy, momentum and angular momentum in classical mechanics, but did not make a systematic connection with geometric invariances. This had been elaborated by Schütz (1897) and by other precursors of Noether: Hamel (1904) who introduced the calculus of variations in that context, Herglotz (1911), Engel (1916) and Kneser (1917) who applied it to the 10 conservation laws due to Galilean and to relativistic invariance, see [13, 15]. But E. Noether was the first to give a general and systematic derivation of conservation laws, starting from invariance of an action principle under Lie algebraic transformations.

This important result of Noether had a curious fate. After initial applause by Klein, Hilbert and others, and some generalization by Bessel-Hagen, came a long freeze. That was caused mainly by the rise of quantum mechanics, which made no use of the Lagrangian formalism. Thus Noether's theorem was essentially forgotten until the early 50's, when covariant Quantum Field Theory (QFT) developed, causing a revival of interest in the Lagrangian formalism, and Noether theorem became important again.

In modern QFT, her theorem appears in particular in the guise of WardTakahashi identities satisfied by the vacuum expectation values of "time-ordered products of fields", (T-products in short), which are the relevant Green functions. In the latter the field operators are ordered from right to left according to 
increasing time, $T \phi_{1}\left(y_{1}\right) \cdots \phi_{n}\left(y_{n}\right):=\phi_{\pi_{1}}\left(y_{\pi_{1}}\right) \cdots \phi_{\pi_{n}}\left(y_{\pi_{n}}\right)$, with $\pi$ a permutation of $\{1, \cdots, n\}$ such that $y_{\pi_{1}}^{0} \geq y_{\pi_{2}}^{0} \geq \cdots \geq y_{\pi_{n}}^{0}$. Take an "internal" symmetry $\left(X_{s}^{\mu}=0\right.$ in the above notations), consider its Noether currents $j_{s}^{\mu}$ and the divergence of its time-ordered product with fields $\left\langle T j_{s}^{\mu}(x) \phi_{1}\left(y_{1}\right) \cdots \phi_{n}\left(y_{n}\right)\right\rangle$. In addition to the explicit divergence which vanishes because of the current conservation, $\partial_{\mu} j_{s}^{\mu}=0$, there is a contribution coming from the implicit Heaviside functions $\theta\left( \pm\left(x^{0}-y_{i_{p}}^{0}\right)\right)$ in the T-product. Then one finds

$$
\begin{aligned}
\frac{\partial}{\partial x^{\mu}}\left\langle T j_{s}^{\mu}(x) \phi_{1}\left(y_{1}\right)\right. & \left.\cdots \phi_{n}\left(y_{n}\right)\right\rangle \\
& =\sum_{i=1}^{n} \delta\left(x^{0}-y_{i}^{0}\right)\left\langle T \phi_{1}\left(y_{1}\right) \cdots\left[j_{s}^{0}(x), \phi_{i}\left(y_{i}\right)\right] \cdots\right\rangle
\end{aligned}
$$

and the equal time commutator on the r.h.s. is the density of the infinitesimal variation of the field $\phi_{j}:\left[j_{s}^{0}(x), \phi_{i}\left(y_{i}\right)\right]_{x^{0}=y_{i}^{0}}=Z_{s}^{i}\left(x, \phi_{i}\right) \delta^{3}\left(\vec{x}-\vec{y}_{i}\right)$. These identities lead to very useful relations between different T-products.

In the case the symmetry is not exact but is "softly broken" and one has a partial conservation of the current $\partial_{\mu} j_{s}^{\mu}(x)=\chi(x)$, with $\chi$ an explicitly known field, the content of the suitably modified identity (5.2) is not void but leads to relations between amplitudes that have been explored in great detail, in particular in the context of weak interactions.

These identities and their various avatars - in particular the Slavnov-Taylor and BRST (Becchi-Rouet-Stora-Tyutin) identities in the framework of gauge theories - play a crucial role at several steps of the study of quantum field theories. They enable one to establish that the renormalization procedure does not jeopardize the symmetries of the original theory; they allow one to prove that conserved currents "do not renormalize" and do not develop anomalous dimensions, thus justifying the notion of universality in "currentcurrent" interactions, see below; they are also used in the derivation of "low energy theorems", see in particular 29.

For completeness, let us mention briefly Noether's second theorem: for an "infinite dimensional group" of invariance (such as diffeomorphisms in General Relativity, or gauge transformations in gauge theories), invariance within a variational principle implies the existence of constraints between the $\Psi_{i}=\delta \mathcal{L} / \delta \phi^{i}$, i.e. identities satisfied independently of the Euler-Lagrange equations of motion. Examples are provided by the contracted Bianchi identities in General Relativity, $D^{\mu} G_{\mu \nu}=0$, where $G_{\mu \nu}=R_{\mu \nu}-\frac{1}{2} g_{\mu \nu} R$, or their analogue $D^{\mu} D^{\nu} F_{\mu \nu}=0$ in gauge theories. Note that, although they are satisfied irrespective of the Euler-Lagrange equations, (4.1) or $\frac{1}{g^{2}} D^{\nu} F_{\mu \nu}=J_{\mu}:=$ $\partial \mathcal{L}_{\mathrm{m}} / \partial A^{\mu}$ respectively, these identities ensure the consistency of the latter, whose right hand sides are covariantly conserved $D^{\mu} T_{\mu \nu}=0$, resp. $D^{\mu} J_{\mu}=0$. 


\section{Invariances in Quantum Mechanics}

With the triumph of Quantum Mechanics, a new paradigm appears in the study of symmetries in physical systems. Through the fundamental papers and books of von Neumann and Wigner, Weyl and van der Waerden [28, 31, [34, 27] at the end of the 1920's, representation theory enters Physics. This is particularly well summarized in Wigner's theorem. With any quantum system is associated a Hilbert space $\mathcal{H}$. States of the system are described by vectors $\Psi$, or more precisely by rays, of $\mathcal{H}$ and "observables" $A$ are self-adjoint operators on $\mathcal{H}$. Then Wigner's theorem [31, 34] asserts

Theorem 6.1 (Wigner). Transformations of a quantum system under a group $G$ are implemented as $\Psi \rightarrow U \Psi, A \rightarrow U A U^{-1}$ with $U$ unitary or anti-unitary and unique up to a phase, satisfying

$$
g, g^{\prime} \in G \quad U(g) U\left(g^{\prime}\right)=U\left(g \cdot g^{\prime}\right) e^{i \omega\left(g, g^{\prime}\right)} .
$$

Thus $U(g)$ gives a projective (up to a phase) representation of $G$.

By "anti-unitary", we mean a unitary antilinear operator, a situation which is encountered in the study of the time reversal operator $T$. Note that the projective nature of the representations is forced upon us by the structure of Quantum Mechanics: rays rather than vectors are the relevant objects.

Among such transformations, invariances are associated with group actions that commute with the dynamics, i.e. with the Hamiltonian

$$
[H, U(g)]=0 \text {. }
$$

But according to Ehrenfest's theorem, the time derivative of any operator (with no explicit time dependence) is given by its commutator with $H, i \hbar \mathrm{d} A / \mathrm{d} t=$ $[H, A]$. Thus (6.1) tells us that any $U(g)$ or any infinitesimal generator of the group action, is conserved: here again, invariances manifest themselves by the existence of conserved quantities. The new feature due to quantum mechanics is that not all conserved quantities are simultaneously observable. If one picks $H$ and a set of commuting operators $U(h)$, ( $h$ in a Cartan torus of $G$ if $G$ is a Lie group), eigenstates of those $U(h)$ have conserved eigenvalues, which are, in the physicists' jargon, "good quantum numbers".

For example, consideration of the group of rotations $\mathrm{SO}(3)$ shows that its infinitesimal generators (i.e. elements of its Lie algebra) are proportional to the components of the angular momentum $\vec{J}$. The latter is thus quantized by the theory of representations of $\mathrm{SO}(3)$. If the system under study is invariant under rotations, one has conservation of $\vec{J}^{2}$ (the Casimir operator) and of one component, say $J_{z}$ : their eigenvalues $j(j+1) \hbar^{2}$ and $m \hbar$ are "good quantum numbers", conserved in the time evolution. States of the system are classified by representations of $\mathrm{SO}(3)$ or $\mathrm{SU}(2)$, the latter appearing because it gives the 
projective (up to a sign) representations of the former, through half-integer spin representations.

As a side remark, we also notice that the distinction between discrete and continuous invariances, that was crucial in classical physics, with only the latter leading to conservation laws, fades away. Conservation of parity - to the extent it is conserved - is expressed by the commutation relation $[P, H]=0$ and implies that the parity of a state is a good quantum number.

This beautiful framework was first applied to the rotation group and its finite subgroups, in conjunction with parity and the symmetric group of permutations. The latter appears in connection with the Pauli principle and the Fermi-Dirac or Bose-Einstein quantum statistics. This resulted in innumerable applications to atomic, molecular and solid state physics: atomic and molecular orbitals, the fine structure of spectral lines of atoms and their splitting in a magnetic or electric field (respectiveley the Zeeman and the Stark effects), the crystal-field splitting and many other effects were analysed by group theoretic methods; selection rules in transitions were shown to be governed by tensor products of representations, etc. See for example [26] for a review, and 22] for an overall presentation of the work of the first actors - Wigner and von Neumann, Heitler and London, Weyl. First applications to particle physics were exploiting rotation, parity and Lorentz invariance in scattering theory. In the latter context, let us cite Wigner's fundamental work on the representations of the Poincaré group [36. For a one-particle state, these representations are fully characterized by two real numbers, which describe the mass and the spin of the particle. But more group theory was soon to come in particle physics and we devote the next section to these new symmetries.

As it is often the case when a new theoretical corpus develops, requiring the learning and the practice of an abstract formalism, not everybody accepted happily this irruption of group theory into physics and there was a certain resistance among some physicists. Some even talked about "the group pest"!..., see [37, 27] p. 165, 22], or the prefaces of [31, 24]. In his preface to the 1959 edition of his book [34, Wigner observes: "It pleases the author that this reluctance [among physicists toward accepting group theoretical arguments] has virtually vanished in the meantime and that, in fact, the younger generation does not understand the causes and the basis for this reluctance."

\section{Invariances in particle physics}

We have seen above that Noether's reciprocal statement enables one to infer the existence of a symmetry group from conserved quantities. This observation has been beautifully illustrated by the discovery of "flavor groups" in particle physics. 
Heisenberg (1932) observing the many similarities of mass and interactions of the two constituents of the nucleus, the nucleons, namely the neutron $n$ and the proton $p$, their electric charge notwithstanding, proposed that they form a 2-dimensional representation of a new $\mathrm{SU}(2)$ group of "isotopic spin", or "isospin" in short. This was an extremely fruitful idea, soon confirmed by the discovery (1947) of the $\pi$ mesons, or pions, coming in three states of charge $\left(\pi^{+}, \pi^{0}, \pi^{-}\right)$, and hence forming a 3 -dimensional representation of this $\mathrm{SU}(2)$ group. Isospin symmetry then predicts relations between scattering amplitudes of nucleons and pions that were well verified in experiments. Later, more instances came with the kaons $\left(K^{+}, K^{0}\right)$, the $\Delta$ resonance $\left(\Delta^{++}, \Delta^{+}, \Delta^{0}, \Delta^{-}\right)$ and others, which form representations of isospin $1 / 2,3 / 2 \ldots$ respectively. This $\mathrm{SU}(2)$ group is a symmetry of hadrons (i.e. of strongly interacting particles), broken by electromagnetic interactions.

In the sixties, the story repeated itself. In view of the newly discovered "strange" particles, Gell-Mann and Ne'eman (1961) proposed the existence of an SU(3) group of (approximate) symmetry of strong interactions. This "flavor SU(3)" group encompasses the previous isospin group SU(2). The argument leading to $\mathrm{SU}(3)$ was that there was experimental evidence of the existence of two independent conserved quantities (isospin and hypercharge or strangeness), hence the group should be of rank 2 . Also there were several observed "octets" (8-dimensional representations) of particles of similar masses and same quantum numbers (baryonic charge, spin, parity), and this pointed to the group $\mathrm{SU}(3)$ which has an 8-dimensional irreducible representation, namely its adjoint representation. This hypothesis was confirmed soon after by the experimental discovery of a particle $\Omega$ completing a 10-dimensional representation, whose mass and quantum numbers had been predicted, and by some other experimental evidence 9]. Associated with the fundamental 3 -dimensional representation of $\mathrm{SU}(3)$ is a triplet of "quarks", $(u, d, s)$ (for up, down and strange), which according to the confinement hypothesis, should not appear as observable particles in normal circumstances 5 . The SU(3) group has been dubbed "flavor" to distinguish it from another "color" SU(3) that appears as the gauge group of "quantum chromo-dynamics" (QCD), the modern theory of strong interactions. To conclude this discussion, let us stress that the flavor $\mathrm{SU}(3)$ group of (approximate) symmetry was more than welcome, in order to put some order and structure in the "zoo" of particles that started to proliferate at the end of the fifties.

This line of thought has proved extremely fruitful, and modern particle physics has seen a blossoming of discoveries structured by the concepts of symmetries and group theory. The previous $\mathrm{SU}(2)$ and $\mathrm{SU}(3)$ groups have been extended to larger flavor groups, in connection with the discovery of new

\footnotetext{
${ }^{5}$ By "normal circumstances" we mean discarding the extreme conditions of the primordial Universe, immediately after the Big Bang, or of the high-energy heavy ion collisions in the laboratory, where a plasma of unconfined quarks may be created.
} 
families of particles, with new quantum numbers, revealing the existence of more species (or "families") of quarks.

The role of symmetries is not limited to strong interactions and the other subatomic forces - electromagnetic and weak - are also subject to symmetry requirements. This was hardly apparent in the early Fermi "current-current" theory of the weak interactions, $\mathcal{L}_{\mathrm{F}}=-\frac{G_{F}}{\sqrt{2}} J^{\mu} J_{\mu}$, but then the V-A pattern à la Gell-Mann-Feynman of the current $J=V-A$, the role of the conservation or partial conservation of currents $V$ and $A$, the Cabibbo angle, etc. were gradually uncovered, see [19, 11] for reviews of these historical developments. This role of symmetries is even more manifest nowadays in the GlashowSalam-Weinberg model of electroweak interactions, see below.

To look for a group invariance whenever a new pattern is observed has become a second nature for particle physicists.

\section{The many implementations of symmetries in the quantum world}

When discussing symmetries in contemporary physics, it is common to distinguish space-time symmetries, discrete or continuous, - rotations and Lorentz transformations, translations, space or time reflections, ...- from "internal" symmetries that act on internal degrees of freedom - charge, isospin, etc. While this distinction may be useful, it should not hide the tight interlacing of these two species of symmetries. For instance, one of the fundamental results in QFT is the CPT theorem (Lüders, Pauli and Bell) which asserts that the product of the charge conjugation $C$ by the space reflection or parity $P$ and time reversal $T$ should be an absolute and uninfringed symmetry of Nature. This is established based on fundamental properties like locality and Lorentz invariance that one expects from any decent theory 256 .

Another distinction between two big classes of symmetries deals with their "global" or "local" character. The isospin SU(2) or the flavor SU(3) symmetries mentioned above are global symmetries, in the sense that the group element describing the transformation is independent of the space-time point where it applies. In contrast, the diffeomorphisms of GR or the gauge transformations of electrodynamics or Yang-Mills theory are local, with the group (or in infinitesimal form, the Lie algebra) element varying from point to point. As we have seen, that distinction was already clearly perceived by Klein and Noether.

\footnotetext{
${ }^{6}$ As pointed out by Yang [38, there is a very intriguing sentence in Weyl's preface to the second edition of his book 31, which seems to indicate that as early as 1930, he foresaw some relation between these three transformations.
} 
It turns out that a quantum symmetry may be realized in a multiplicity of ways, namely

- as an exact symmetry, e.g. in the global U(1) symmetries associated with charge or baryonic number conservation, or in the local gauge invariances of quantum electrodynamics and of quantum chromodynamics (QED, $\mathrm{QCD})$;

- as an explicitly broken symmetry: this is the case with isospin $\mathrm{SU}(2)$ broken by electromagnetism, or flavor $\mathrm{SU}(3)$, which is an approximate symmetry, broken by the strong interactions themselves. This is also the case with parity, the space reflection $P$ mentioned above, which is explicitly broken by weak interactions, as discovered by Lee and Yang (1956) and as now implemented in the Standard Model;

- as a spontaneously broken symmetry. This refers to the following situation: in a physical system a priori endowed with a certain symmetry, the state of minimum energy, called the ground state or the vacuum depending on the context, may in fact be non invariant. This is a very common and fundamental phenomenon, which is familiar from the case of ferromagnetism: in a ferromagnet in its low-temperature phase, the magnetic moments of the individual atoms, although subject to a rotation invariant interaction, pick collectively a direction in which they align on average, thus giving rise to a macrocopic magnetization that breaks the rotation invariance of the whole system. This is accompanied, if the broken symmetry is continuous, by the appearance of massless excitations or particles, associated with the possibility of continuously rotating the ground state at a vanishing cost in energy. These excitations are the Nambu-Goldstone particles. In the variant in which the symmetry is only approximate, and in the neighbourhood of a spontaneously broken phase, one expects the would-be Nambu-Goldstone bosons to be not strictly massless but of low mass;

- as a spontaneously broken gauge symmetry: a global symmetry is spontaneously broken but the resulting theory maintains an exact gauge invariance. Then, and this is the essence of the Brout-Englert-Higgs (BEH) mechanism, the Nambu-Goldstone excitations do not appear as real particles, and instead give rise to additional polarization states of some vector fields and to masses of the corresponding particles. This is a crucial step in the edification of the electro-weak sector of the Standard Model, and the successive discoveries of massive vector particles (the $W^{ \pm}$and $Z^{0}$ ) and lately, of a candidate for the relique massive scalar boson at CERN, seem to corroborate this model;

- anomalously, which means through a breaking of a classical symmetry by quantum effects. Examples are provided by the realization of some chiral symmetries of fermions, which act separately on the left-handed and 
right-handed components of these particles. Conversely, in the Standard Model of particle physics, where the assignments of representations are different for different chiralities, it is essential that anomalies cancel, see below;

- with supersymmetry: that ordinary Lie groups and algebras could be extended to accommodate anticommuting (Grassmannian) elements has been known and well studied since the seventies. To this date we have not seen any direct manifestation of supersymmetry in the laboratory. But the idea has been so amazingly fruitful in establishing new results and new connections between different fields that it will undoubtedly remain in the physicist's toolbox;

- as quantum symmetries, or "quantum groups", a misnomer for "quantum" deformations of Lie algebras or, more generally, for Hopf algebras. These have not yet manifested themselves in the context of particle physics, but are determinant in the discussion of quantum integrable models and in their applications to many systems of condensed matter physics in low dimension;

... and this list is certainly non exhaustive.

It is truly remarkable that Nature makes use of all these possible implementations of symmetries.

Let us illustrate these various possibilities on a few examples coming from modern physics. Our presentation will be extremely sketchy, as each topic would deserve a separate monograph...

Example 1: "Linear/non linear sigma models" may be regarded as Klein's most direct heirs in the context of QFT.

In the simplest possible case, consider a field $\phi$ defined on $\mathbb{R}^{d}$ and taking its values in $\mathbb{R}^{n}$ or $\in S^{n-1}$ and write a Lagrangian in the form

$$
\mathcal{L}=\frac{1}{2}(\partial \phi, \partial \phi)-V((\phi, \phi))
$$

where $($,$) denotes the \mathrm{O}(n)$ invariant bilinear form. The invariance group of that Lagrangian is obviously $\mathrm{O}(n)$, and the field $\phi$ transforms according to a linear representation or to a non-linear realization, depending on the case $\mathbb{R}^{n}$ resp. $S^{n-1}$. According to Noether's theorem, there are $\frac{1}{2} n(n-1)$ independent conserved quantities at the classical level. Using the corresponding Ward identities (5.2), one verifies that the symmetry is preserved by quantum corrections. This was first set up by Gell-Mann and Lévy (1960) in the case $n=4$, in their investigation of the partial conservation of the "axial current" in weak intercations [8], and involved the fields of pion particles $\pi^{ \pm}, \pi^{0}$ and of a hypothetical field $\sigma$, whence the name given to the model; this original model had thus a (softly and spontaneously broken) $\mathrm{O}(4)$ symmetry. 
This may be generalized to a field $\phi$ taking its values in $\mathcal{M}$, a Riemannian manifold with isometries. Now in any of these sigma models, the natural questions to ask are

- how is the symmetry realized, as an exact, explicitly broken, or spontaneously broken symmetry?

- how is the symmetry preserved by renormalization? This is where use has to be made of Noether currents and Ward identities;

- what are the physical consequences: are there Goldstone particles, or "almost Goldstone" particles (like the pion of low mass)? is there a dynamical generation of mass? is the theory scale or conformally invariant? and so on, and so forth.

Sigma models have been extensively used with all kinds of manifolds and groups in particle physics and cosmology, in statistical mechanics and solid state physics. For example they appear as effective low-energy theories for various phenomena in condensed matter, describing membranes, surface excitations, order parameters, ...; but also in string theory - again in a low energy limit -, based on ordinary manifolds or generalized geometries à la Hitchin. The study of non compact and/or supersymmetric sigma models is a currently very active subject, for its applications running from condensed matter to string theory.

These sigma models also constitute a mine of mathematical problems. For instance, particular cases with $V=0$ are studied for their own sake, in Riemannian geometry, under the name "harmonic maps".

Example 2: The Standard Model of particle physics has a symmetry group $\mathrm{SU}(3) \times \mathrm{SU}(2) \times \mathrm{U}(1)$, with three gauge groups realized in a completely different way.

The $\mathrm{SU}(3)$ color (gauge) symmetry of QCD is an exact invariance, and this is believed to be of crucial importance for quark confinement. On the other hand $\mathrm{SU}(2) \times \mathrm{U}(1)$, the gauge group of weak isospin and weak hypercharge, is spontaneously broken down to an exact $\mathrm{U}(1)$, the gauge symmetry of ordinary electrodynamics. As mentioned above, a relique of the $\mathrm{BEH}$ mechanism at work in this spontaneous breaking should be a spin 0 boson, a good candidate of which has just been observed at CERN.

The absence of anomalies in the Standard Model, crucial for the consistency of the theory, relies on a remarkable matching between families of leptons and of quarks: for both types of particles three "generations" are known at this time

$$
\left(e, \nu_{e}\right),\left(\mu, \nu_{\mu}\right),\left(\tau, \nu_{\tau}\right) \longleftrightarrow(u, d),(c, s),(t, b),
$$

and anomalies cancel within each generation [1].

On top of the gauge pattern, there are other $\mathrm{SU}(2)$ and $\mathrm{SU}(3)$ groups at work: the flavor $\mathrm{SU}(2) \subset \mathrm{SU}(3)$ broken symmetries discussed above. In another 
vein, a scenario which has been contemplated - and in fact studied in great detail - but does not yet seem to be borne out by experiments is that this Standard Model is in fact a subsector of a larger supersymmetric extension.

Example 3: Quantum integrable systems and Quantum Groups Consider the spin $\frac{1}{2} \mathrm{XXZ}$ quantum chain: this is a quantum system of $N$ spins whose interactions are described by the Hamiltonian

$$
H=\sum_{i=1}^{N} S_{i}^{x} S_{i+1}^{x}+S_{i}^{y} S_{i+1}^{y}+\Delta S_{i}^{z} S_{i+1}^{z}+\text { boundary terms }
$$

acting in $\left(\mathbb{C}^{2}\right)^{\otimes N} . \Delta$ is an anisotropy parameter in spin space. It was first introduced for $\Delta=1$ by Heisenberg (1928) as a model of ferromagnetism. This is known to be a quantum integrable system after important contributions by Bethe, Lieb and Sutherland, Yang and Yang, Gaudin, Baxter, Faddeev and many others. For $\Delta=1$, (and no boundary term), it exhibits $\mathrm{SU}(2)$ invariance. For $\Delta \neq 1,|\Delta|<1$, it has a deformed symmetry $U_{q} s l(2)$ ("quantum $\mathrm{SU}(2)$ "), where $q=e^{i \alpha}, \Delta=\cos \alpha$ [20, or an affine quantum $U_{q} \widehat{s l}(2)$ [7, depending on the boundary conditions. Recent progress on the computation of correlation functions of the XXZ chain and on its connections with problems of combinatorics have been made possible by representation theoretic considerations.

Other recent advances in the context of integrable gauge theories and the AdS/CFT correspondence also rely to a large extent on representation theory of quantum algebras.

Example 4: Conformal invariance. The last fifteen years of the previous century have witnessed rapid progress in our understanding of quantum field theories in low dimension. In $2 \mathrm{~d}$, conformal invariant field theories (CFTs) have experienced a spectacular development, with a huge number of exact results and applications to critical phenomena and to string theories, thus writing a new chapter of non-perturbative quantum field theory. For the largest part, this progress was made possible by advances at the end of the seventies in the representation theory of infinite dimensional Lie algebras - Virasoro, affine Lie algebras and their cousins - that are the relevant symmetries of CFTs. For a review, see for example [5]. There one sees once again the close ties between symmetries, group theory and their physical implications. 


\section{Conclusions}

We have seen that symmetries and group theory play an essential role in modern physics. Their role is:

- to dictate the possible form of interactions on geometrical grounds: the cases of General Relativity or of gauge theories are exemplary in that respect; but one may also quote non-linear sigma-models, in which the form of the Lagrangian is prescribed by the geometry of the manifold and the isometries are playing a key role;

- to predict: more invariance means less independence, implying relations between different phenomena, selection rules, a priori determination of multiplicities, etc., as illustrated by scores of examples in atomic, molecular, solid state and particle physics; and to organize a wealth of data, of particles, of phenomena: we have seen that representation theory is instrumental in this undertaking;

- to protect in the quantization (and renormalization). Once again, take the example of a gauge theory. Were its symmetries broken by quantum effects (ultraviolet divergences, anomalies), the theory would lose most of its predictive power or even become inconsistent. So we have a self-consistent picture, where symmetry implies constraints (in the form of Ward identities), that in turn guarantee that symmetry is preserved by quantization. This scheme is implemented recursively in the perturbative construction of gauge theories.

The study of groups and of representation theory is now part of the education of a modern physicist. Some domains of representation theory - of superalgebras, of quantum groups and of infinite dimensional algebras - have developed recently thanks to the incentive of physical applications.

Could a unified theory based on geometry and embracing all fundamental interactions including gravitation be constructed? That was Einstein's dream, this is still regarded today as the Holy Grail by many people, string theorists among others.

We have emphasized the many possible implementations of symmetries in (quantum) physics. We have also stressed that not only the nature of the symmetry group but also the scheme of its breaking, and the residual subgroup of symmetry, are determinant. In that respect, we are still living in the legacy of Klein and Curie... 


\section{Acknowledgements}

It is a pleasure to thank the organizers, Lizhen Ji and Athanase Papadopoulos, for inviting me to this meeting, and Michel Bauer, Matteo Cacciari, Robert Coquereaux, Yvette Kosmann-Schwarzbach and Raymond Stora for several useful suggestions on a first draft of the manuscript.

\section{References}

[1] C. Bouchiat, J. Iliopoulos and P. Meyer, An anomaly-free version of Weinberg's model, Phys. Lett. 38B (1972) 519-523

[2] J.J. Burckhardt, Die Bewegungsgruppen der Kristallographie, Birkhäuser, 1966

[3] P. Curie, Sur la symétrie dans les phénomènes physiques, symétrie d'un champ électrique et d'un champ magnétique, Journ. de Physique 3 393-415 (1894)

[4] O. Darrigol, The Genesis of the theory of relativity, Séminaire Poincaré 1: 122 (2005);

O. Darrigol in Einstein aujourd'hui, EDP Sciences 2005

[5] P. Di Francesco, P. Mathieu and D. Sénéchal, Conformal Field Theory, Springer 1997

[6] A. Einstein to V. Bargmann, as quoted in Pais [18, page 152

[7] M. Jimbo and T. Miwa, Algebraic Analysis of Solvable Lattice Models, (NSFCBMS Regional Conference 1993), AMS 1995

[8] M. Gell-Mann and M. Lévy, The axial vector current in beta decay, Nuovo Cim. 16 (1960) 705-726

[9] M. Gell-Mann and Y. Ne'eman, The Eightfold Way, Benjamin 1964

[10] W. Heisenberg, Über den Bau der Atomkerne, Z. für Physik 77 (1932) 1-11

[11] J. Iliopoulos, Symmetries and the weak interactions, talk given at the Cabibbo Memorial Symposium, Rome 2010, http://lanl.arxiv.org/abs/1101.3442

[12] T. Janssen, Crystallographic Groups, North Holland 1973

[13] H. A. Kastrup, The contributions of Emmy Noether, Felix Klein and Sophus Lie to the modern concept of symmetries in physical systems, in Symmetries in Physics (1600-1980), ed. M. G. Doncel, Universitat Autònoma de Barcelona, 1987, pp. 113-163

[14] F. Klein, Über die geometrischen Grundlagen der Lorentzgruppe, Jhrber. $d$. d. Math. Vereinig. 19 (1910) 287, reprinted in Gesammelte Mathematischen Abhandlungen, vol I, Springer 1921, 533-552

[15] Y. Kosmann-Schwarzbach, The Noether Theorems, Invariance and Conservation Laws in the Twentieth Century, Springer (2006) 
[16] L. Michel, Symmetry, invariants, topology. IV Fundamental concepts for the study of crystal symmetry, Physics Reports 341 (2001) 265-336

[17] E. Noether, Invariante Variationsprobleme, Nachrichten von der Königlichen Gesellschaft der Wissenschaften zu Göttingen (1918) 235-257

[18] A. Pais, Subtle is the Lord ..., The Science and the Life of Albert Einstein, Oxford Clarendon Press, 1982

[19] A. Pais, Inward Bound: Of Matter and Forces in the Physical World, Oxford University Press (1986)

[20] V. Pasquier and H. Saleur, Common structures between finite systems and conformal field theories through quantum groups, Nucl. Phys. B 330 (1990), $523-556$

[21] F.C. Phillips, An introduction to Crystallography, Longman 1963

[22] E. Scholz, Introducing groups into quantum mechanics, Historia Mathematica 33 (2006), 440-490, http://lanl.arxiv.org/math.HO/0409571

[23] M. Senechal, Quasicrystals and geometry, Cambridge University Press 1995

[24] S. Sternberg, Group theory and physics, Cambridge Univ. Pr. 1994

[25] R.F. Streater and A.S. Wightman, PCT, Spin and Statistics and All That, Benjamin 1964

[26] M. Tinkham, Group Theory and Quantum Mechanics, McGraw-Hill 1964, Dover 2003

[27] B. van der Waerden, Die gruppentheoretische Methode in der Quantenmechanik, Springer 1932

[28] J. von Neumann and E. Wigner, Atomic wave functions with spinning electrons, Z. für Physik 47 (1928) 203; 49 (1928) 73

[29] S. Weinberg, The Quantum Theory of Fields, vol. II, Cambridge Univ. Pr. 1996

[30] H. Weyl, 1918, reprinted in Gesammelte Abhandlungen, vol II, K. Chandrasekharan ed., Springer, p 29

[31] H. Weyl, Gruppentheorie und Quantenmechanik, Hirzel (Leipzig) 1928); The Theory of Groups and Quantum Mechanics, Dutten (New York) 1931, Dover 1950

[32] H. Weyl, Z. Physik 56 (1929) 330, reprinted in Gesammelte Abhandlungen, vol III, K. Chandrasekharan ed., Springer, p 245

[33] H. Weyl, Symmetry, Princeton University Press 1952

[34] E. Wigner, Gruppentheorie und ihre Anwendung auf die Quantenmechanik der Atomspektren, F. Vieweg \& Sohn, 1931; Group Theory and its Applications to the Quantum Mechanics of Atomic Spectra, Academic Press 1959

[35] E. Wigner, On the Consequences of the Symmetry of the Nuclear Hamiltonian on the Spectroscopy of Nuclei, Phys. Rev. 51 (1937) 106-119 
[36] E. Wigner, On Unitary Representations of the Inhomegeneous Lorentz Group, Ann. Math 40 (1939) 149-204

[37] E. Wigner, Oral transcript of an Interview with Dr. Eugene Wigner By Lillian Hoddeson, Gordon Baym and Frederick Seitz, (1981), Center for History of Physics of the American Institute of Physics, http://www .aip.org/history/ohilist/4965.html

[38] C.N. Yang, Herman Weyl's contribution to Physics, in Herman Weyl, 18851985, Centenary lectures, K. Chandrasekharan ed., Springer 1986

[39] C.N. Yang and R. Mills, Conservation of Isotopic Spin and Isotopic Gauge Invariance, Phys. Rev. 96 (1954) 191-195 